\title{
Methods for analysis of the cancer microenvironment and their potential for disease prediction, monitoring and personalized treatments
}

\author{
Carl-Magnus Clausson, Ida Grundberg, Irene Weibrecht, Mats Nilsson and Ola Söderberg ${ }^{*}$
}

\begin{abstract}
A tumor does not consist of a homogenous population of cancer cells. Therefore, to understand cancer, the tumor microenvironment and the interplay between the different cell types present in the tumor has to be taken into account, and how this regulates the growth and survival of the cancer cells. To achieve a full picture of this complex interplay, analysis of tumor tissue should ideally be performed with cellular resolution, providing activity status of individual cells in this heterogeneous population of different cell-types. In addition, in situ analysis provides information on the architecture of the tissue wherein the cancer cells thrive, providing information of the identity of neighboring cells that can be used to understand cell-cell communication. Herein we describe how padlock probes and in situ PLA can be used for visualization of nucleic acids and protein activity, respectively, directly in tissue sections, and their potential future role in personalized medicine.
\end{abstract}

Keywords: PLA, Padlock probes, Tumor microenvironment, Personalized medicine, Diagnosis, Prognosis

\section{The cancer cell}

Progression from a normal cell to a cancer cell usually requires several genetic alterations including overexpression or alterations of oncogenes and loss of tumor suppressor genes [1]. Whole genome sequencing studies reveal that hundreds of genes are more or less frequently mutated in cancer [2]. Most mutated genes are only altered in a small subset of patients with a particular cancer disease, while only a few genes are commonly mutated. However, collectively rare mutations make up a large proportion of the mutational landscape of cancer. While the DNA sequence acts as a blueprint in the molecular assembly of an individual, the functionally active components of a cell are RNA and proteins. Analysis of RNA and protein are therefore needed to determine to what extent the genetic information is utilized to promote growth and survival of cancer cells. However, the question is whether genetic alterations propagating into expressed RNA and proteins are the sole factor causing and sustaining cancer, and how much this is influenced

\footnotetext{
* Correspondence: ola.soderberg@igp.uu.se Department of Immunology, Genetics and Pathology, Science for Life Laboratory, Rudbeck Laboratory, University of Uppsala, S-751 85, Uppsala, Sweden
}

(c) 2012 Clausson et al; licensee BioMed Central Ltd This is an open access article distributed under the terms of the Creative Commons Attribution License (http://creativecommons.org/licenses/by/2.0), which permits unrestricted use, distribution, and reproduction in any medium, provided the original work is properly cited. by epigenetic alterations [3], or if there are additional external factors. To what extent do cells determine their own fates?

\section{Cancer and microenvironment}

For all multicellular organisms intercellular communication is essential to ensure proper organization of individual cells in tissues and organs. The signals transferred between cells provide information that determines the fate of the recipient cells, regulating growth, survival and differentiation. In order to decide to which signals they should respond, cells express different panels of receptors that subsequently will relay the signals, via intricate networks of protein-protein interactions where the activity status of the individual proteins are regulated by post-translational modifications (PTMs). The observed genetic and epigenetic alterations in cancer cells act to promote sustained growth and survival and to overcome the growth restraints from the surrounding cells. The extent of the interplay between cell types, and the regulatory effects from the neighboring cells on the phenotype of a cancer cell, is maybe best exemplified by teratomas. When teratoma cells are introduced into blastocysts of pseudo-pregnant mice they will normalize their phenotype and generate a 
mosaic that will be tumor free $[4,5]$. When teratoma cells are instead transplanted into the sides of 129 /SV mice, they will form tumors. In fact, transplanting induced pluripotent stem cells (iPSC) into mice to form teratomas is presently used as a step for assessing the validity and viability of iPSC [6-8].

While the normal cells surrounding cancer cells sometimes fail to prevent the cancer from proliferating, this prevention seemingly succeeds more often than it fails as dormant and unnoticed cancer are developed throughout life [9]. This was indicated already in 1934 when small carcinoma was discovered at surprisingly high levels $(14 \%)$ in prostates of individuals exceeding 50 years of age having died from unrelated causes [10]. The prevalence was discovered to be even higher (34\%) in a later study examining the entire prostate glands of even younger males [11]. Apparently the pathogenic transformation of a cell into a carcinogenic state is not enough for full-blown tumor growth. Functions external to the cancer cell must somehow influence its capability to proliferate. Thus, properties of the microenvironment have been gaining interest when trying to understand what activates carcinoma into proliferation. It is now evident that cancerous tumors are not the homogeneous collections of cells they were once thought to be, but consists of several types of cell subpopulations (reviewed by Hanahan and Weinberg [12]). These are cancer cells, cancer stem cells, endothelial cells, pericytes, immune inflammatory cells, cancer-associated fibroblasts, and stem and progenitor cells of the tumor stroma [12]. Together they make up the tumor microenvironment but they have different origins, with the cancer cells acting as tumor initiators. The stem and progenitor cells of the tumor stroma, the immune inflammatory cells and the bone marrow-derived vascular progenitor cells are all recruited from circulation or neighboring tissues. This is true also for the cancer-associated fibro-blasts, which can be recruited myofibroblasts or reprogrammed variants of normal tissue-derived fibroblastic cells. Inflammatory cells can be attracted to the tumor by cancer cells which sometimes undergo necrosis, instead of healthy apoptosis $[13,14]$, creating an inflammatory environment that can both antagonize and promote the tumor growth, and in this light tumors have been described as wounds that never heal $[15,16]$. Formation of vasculature is regulated by the balance between secreted pro- and anti-angiogenic factors, supporting the cancer cells with nutrients and oxygen. However, the tumor vessels display abnormal organization that impairs transport of nutrients and oxygen, and facilitates migration of cancer cells [17]. The shuttling of cancer cells between the vascular system (i.e. circulating tumor cells (CTC)) and the tumor tissue has been suggested as an important event (i.e. cancer selfseeding) that promotes the growth of a tumor, as the cancer cells are adapted to the local microenvironment [18]. Some of the CTC will fail in the homing to the local microenvironment and will end up in circulation, where they may colonize other sites, supported by interactions with leukocytes that promote extravasation and survival, giving rise to metastasis $[19,20]$. Immune cells and bonemarrow derived progenitor cells have also been described to promote angiogenesis in tumors [17,21]. Normal stromal fibroblasts are now recognized as being able to induce carcinomas through paracrine signaling with several growth factors [22]. Mesenchymal stem cells can be recruited to tumors and have been observed to differentiate into several cell types in the tumor stroma, and to exert both tumor promoting and suppressing functions in contradicting studies [23]. The concept of cancer stem cells has been widely used during the past few decades to explain different phenotypes and abilities to generate tumors within tumors. An alternative explanation to such events is that the cancer cells experience high plasticity and that differences observed are contingent on the position of the cells in the microenvironment [24,25], to which they adapt to by epigenetic reprogramming, i.e. epithelial-mesenchymal transition (EMT) and mesenchymal-epithelial transition (MET). This is a not yet fully understood program comprising the transition of epithelial cells into cancerous states of varying degrees [12]. Hypoxia or overexpression of hypoxia-inducible factor$1 \alpha($ HIF- $1 \alpha)$ has been shown to promote EMT and a metastatic phenotype [26], suggesting that poor or deficient vascularization of a tumor might induce cancer stem cell properties. The transition into a mesenchymal phenotype is accompanied by epigenetic alterations that activate mesenchymal genes while expressions of epithelial genes are suppressed. To allow repetitive transitions between epithelial and mesenchymal phenotypes bivalent modifications of the promoters by H3K4 methylation and H2K27 trimethylation will keep genes downregulated during EMT, but poised for activation in subsequent MET [27].

It seems that the tumor microenvironment is highly intricate, being made up from numerous cell types all affecting each other in both pathological and normal manners. How may we untangle these interactions among cells and their internal constituents? What methods are needed?

\section{Requirements on analytical methods}

The deregulation of signals in cancer cells, promoting growth and survival to later form tumors, can originate from several levels; the DNA sequence, the epigenetic status, the degree of gene and protein expression, and also protein activity (interactions and post-translational modifications (PTMs)). To obtain a coherent view of a cell, analysis should ideally be performed at several 
levels in the same cell. In cancer research, xenografts and cell lines are commonly used as cancer models in the place of primary tumor material-a limited resource, also complicated by heterogeneity in composition and variation between samples. However, the usage of cancer models involves artifacts. Concerning xenografts, the mouse stroma may not support the growth of a human tumor. For cell lines, normally grown in 2D mono cultures on plastic support and having almost unlimited supply of nutrients and oxygen, the adaptation (epigenetic alterations) and evolution (selection of novel genomic aberrations) caused by in vitro culturing, can severely influence the phenotype of the cells $[28,29]$. However, there are areas of research where cell lines, being homogenous populations and unlimited resources, are suitable. One is in detailed studies of cell signaling kinetics. But one needs to be careful drawing conclusions from cell lines as representatives of tumors in vivo, as the activity status of a cancer cell in vivo will be dependent on where in a tumor it resides as this will influence its exposure to signals, nutrients and oxygen. The emerging view of the heterogeneous cancer microenvironment highlights the need for single cell analysis. Studies of the tumor microenvironment thus need to be performed in clinical tissue samples, which closely represent in vivo states. There are then two alternative approaches: either to isolate the cells of interest and perform the analyses in vitro or perform the analyses in situ, keeping the architecture intact. By micro-dissecting populations of cells, standard analytical methods may be used. However, sensitivity demands increase with decreasing amount of obtained material. In situ analysis on the other hand targets the analyte directly in its normal location, omitting the need to collect cells and purify nucleic acids or proteins. For in situ analysis the available methods are few, the most common ones are fluorescence in situ hybridization (FISH) for analyzing nucleic acids and immunohistochemistry (IHC) for protein detection. These methods are mainly based on the use of probes such as oligonucleotides or antibodies and as a consequence thereof, the selectivity becomes limited due to the inherent crossreactivity of the probes that may generate false positive signals. We will continue with an in depth description of a few in situ methods developed in our research groups that overcome this problem by requiring multiple criteria to be fulfilled for a positive identification, providing single-nucleotide discrimination for detection of nucleic acid and the possibility to visualize the active fraction of a protein, by targeting protein interactions or PTMs. Moreover, simultaneous detection of different biomolecules in situ will help in the understanding of the complex function and regulation of cells. An optimal tool would have the ability to visualize different positions in a signaling pathway and measure phenotypic variations, e.g. expression, within to achieve a more complete picture over what is going on inside a single cell.

\section{Visualization and genotyping of nucleic acids}

Detection of nucleic acids by hybridization of FISH probes [30] has been used for decades but lacks the ability to discriminate between closely similar sequences. To increase the selectivity over what the base pairing of a single probe provides, padlock probes were developed [31]. These are linear oligonucleotides of approximately 70 to 100 nucleotides in length with target-complementary 5'- and 3'- ends which constitute dual target recognition when both probe arms must hybridize correctly to the target. When padlock probes hybridize to their correct target the ends of the padlock probe are brought together in a head to tail orientation, with only a nick in between. The nicks can be sealed by a DNA ligase to create circles that are locked onto the target strands as padlocks [31]. This nick ligation will only occur if there is a perfect match between probe and target at the ligation junction, leaving mismatched probes unligated. Padlock probes were recognized early on for being useful for in situ analysis and the first published application demonstrated genotyping of centromeric sequences [32] using hapten or fluorescence labeled probes. However, with this approach single molecules could not be detected because of high background from unspecifically bound probes. To be able to visualize individual padlock probes, we later used rolling circle amplification (RCA) with the probes as templates. RCA is an isothermal amplification technique of circular DNA molecules that creates long single-stranded DNA molecules with tandem repeats complementary to the original circles [33], here being the padlock probes. The contiguous RCA products will by nature collapse into micrometer-sized DNA-bundles. By targeting the RCA product with fluorophore-labeled oligonucleotides, these can be visualized as bright objects easily distinguishable over background fluorescence [34]. The RCA also selects for circularized padlock probes, as only ligated probes can be amplified, which further enhances the inherent selectivity of the assays. To facilitate the binding of the padlock probe to its target sequence, the DNA needs to be enzymatically prepared in situ, to create a single stranded stretch. This is achieved by combining restriction enzyme digestion with an exonuclease step for target preparation. The free 3'end of the target strand can then be utilized to prime the RCA and it is thus important that this is located close to the bound padlock probe, creating an RCA product that will be an elongation of the targeted sequence (Figure 1). The method was first described for genotyping of mitochondrial DNA (mtDNA) [35]. By having two padlock probes, one for each genotype (designed with different 


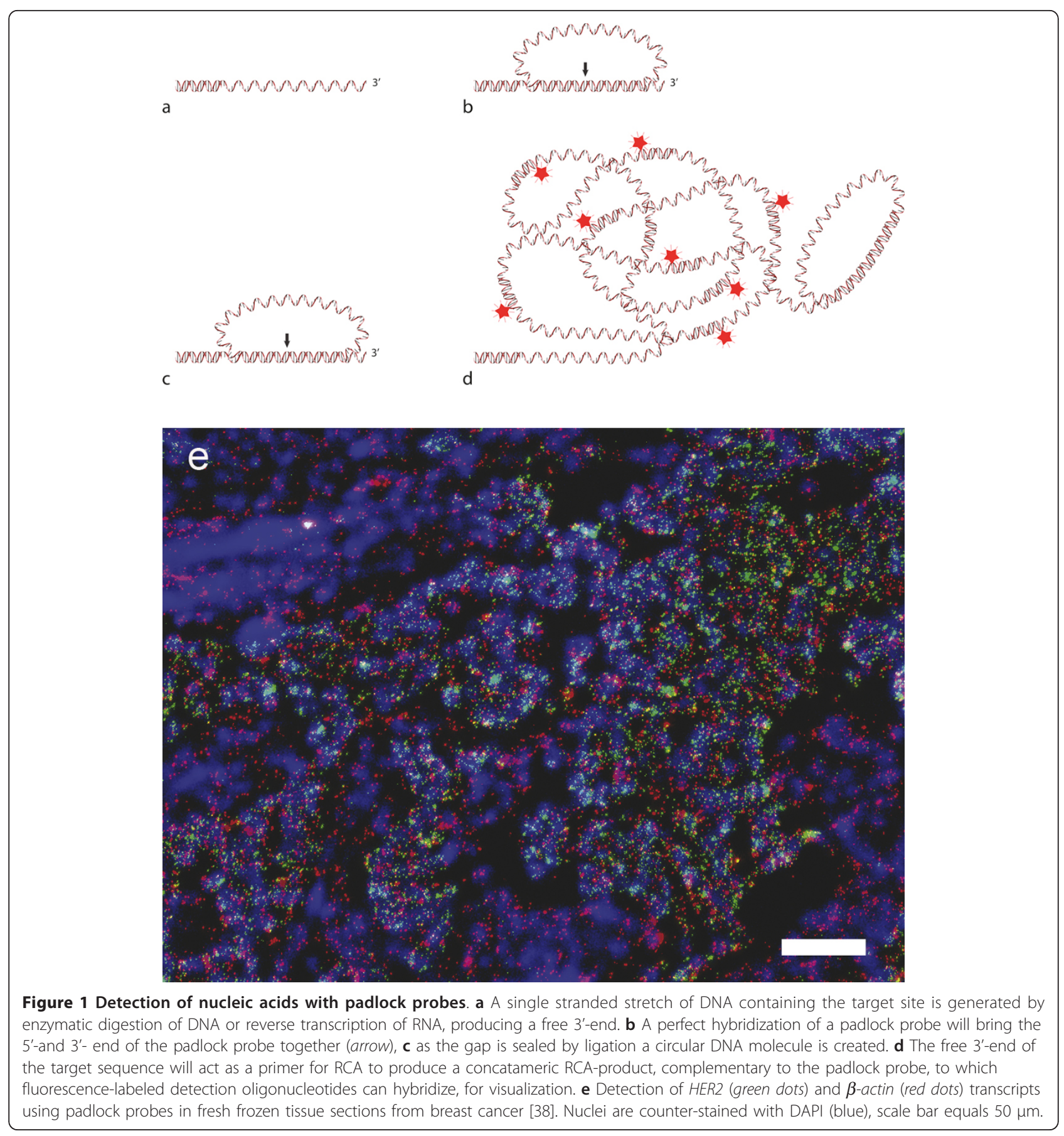

detection sequences), the wild-type genotype was easily distinguished from the mutant in both homo- and heteroplasmic cell lines as well as in fresh-frozen tissue sections [35].

Genotyping of transcripts and gene expression profiling using padlock probes and RCA has been of great interest. However, direct RNA-templated ligation of DNA padlock probes have been shown to be rather inefficient $[36,37]$. We circumvented the limitation of poor ligation efficiency on RNA molecules with a conversion of the mRNA into cDNA [38]. In brief, the method is initiated by in situ reverse transcription using LNA-containing primers, followed by digestion of the mRNA part of the mRNA/cDNA duplex for creation of a singlestranded target. Padlock probes are thereafter hybridized to the cDNA transcripts and circularized upon absolute complementarity to the correct targets and finally, RCA is primed from the cDNA to create visible signals, as 
described above. LNA-modified oligonucleotides have been widely used in several in situ hybridization studies and are known to increase hybridization affinities towards DNA and RNA [39,40]. In addition to substantial improvement of the reverse transcription step, due to increased hybridization efficiency, the LNA-containing cDNA primers also protect the RNA from breakdown by RNase $\mathrm{H}$ [41] - a feature herein utilized to anchor the cDNA molecule to the mRNA throughout the whole procedure. Padlock probes will not just yield information about the genotype of the transcript, but will also provide information on target localization in the tissue and cell. The approach has sufficient resolution to detect point-mutations, splice-variations, and fusion transcripts. Another advantage of analysis at the RNA level, which is closer to the phenotype than DNA, is that it provides information about whether the mutation is expressed or over-expressed and whether the wild-type allele is functionally lost (expressed loss-ofheterozygosity). Thus, this RNA-based assay will also capture effects of promoter and enhancer mutations, epigenetic alterations caused by large chromosomal rearrangements and miRNA effects on transcription.

\section{Visualizing protein interactions and post- translational modifications}

The activity status of proteins is in most cases regulated by PTMs, such as phosphorylation, which will cause structural changes in the proteins and thereby expose catalytic sites or promote interactions with other proteins. For localized detection of protein interactions, double-staining of proteins with IHC is commonly used. Employing two binders targeting different proteins and being differently labeled, the co-localized observation of these infers interaction. However, the resolution of a regular fluorescence microscope is 200-350 $\mathrm{nm}$ [42], which is arguably far too inferior for a positive identification of interacting molecules in a co-localization assay. One approach to improve the detection of colocalized antibody-coupled fluorophores is to utilize the Förster resonance energy transfer (FRET) that occurs between fluorophores with compatible excitation and emission spectra. The phenomenon requires short distances, below $10 \mathrm{~nm}$, and is dependent on the orientation of the fluoro-phores [43]. For detection of protein interactions in tissue sections the fluorophores needs to be coupled to antibodies targeting the interacting pair of proteins $[44,45]$. However, the method is currently hampered by low quantum yield, tissue autofluorescence and over bleeding between fluorophores, making detection of low abundant protein interactions challenging.

To obtain a method that retains the requirement for proximal binding of antibodies to interacting proteins in order to create a signal, as for FRET, but to also provide potent signal amplification, the in situ proximity ligation assay (in situ PLA) was developed [46]. The method utilizes the distance constraints for ligation of two DNA molecules into a reporter molecule and employs PLAprobes which are chimeric molecules consisting of antibodies, for recognizing the protein of interest, equipped with conjugated single stranded oligonucleotides, for subsequent detection purposes. Proximal binding of pairs of PLA-probes to the same target will position the oligonucleotides in close vicinity of each other, which guide the hybridization of two subsequently added circularization oligonucleotides that upon ligation will form a circular DNA molecule. The formation of this DNA circle will thus be strictly dependent on, and will function as a reporter for, the proximal binding of a pair of PLAprobes. In analogy to what is previously described for the padlock probes, the proximity-dependent DNA circle can then be used as a template for RCA, primed by a free 3'end on one of the PLA-probes, to create an RCA product that will be an elongation of the PLA-probe. The RCA products are visualized with detection oligonucleotides and will be localized close to the protein interaction of interest as they are attached to the PLA probe, which still binds its protein target (Figure 2). The method may be used both to detect protein complexes, by targeting two interacting proteins $[46,47]$, and to determine post-translational modifications of a protein, targeting the core protein and a modified epitope [48]. A big advantage is that the bright signals enable detection in formalin-fixed paraffin-embedded tissue sections [49], which commonly suffers from high autofluorescence. To circumvent autofluorescence entirely, it is possible to replace the fluorophore of the detection oligonucleotide with an enzyme, providing an assay for protein interaction analysis by regular brightfield microscopy [50].

Although the distance requirement to generate an in situ PLA signal is theoretically within $\sim 20 \mathrm{~nm}$ (calculated from the size of an antibody and length of the nucleotides), the RCA product produced has a diameter of approximately $1 \mu \mathrm{m}$. While the size of the RCA products provides for easy detection, due to the high number of repetitive targets for fluorophore-labeled detection oligonucleotides, it also limits the number of RCA products that can be detected in a cell. A few hundred RCA products per cell are often enough to cause saturation, when discrete objects can no longer be defined. To increase the limited dynamic range it is possible to use a mix of different circularization oligonucleotides, with different sequences for detection oligonucleotides, such that the concentration ratio of the added circularization oligonucleotides will then be reflected among the RCA products. In practice, the effect is that when one type of RCA product labeled with a distinct detection oligonucleotide saturates the area of analysis, it is possible to instead view 
Clausson et al. EPMA Journal 2012, 3:7

Page 6 of 9

http://www.epmajournal.com/content/3/1/7

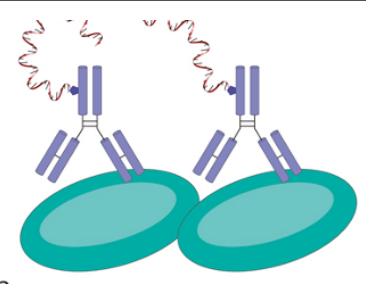

a

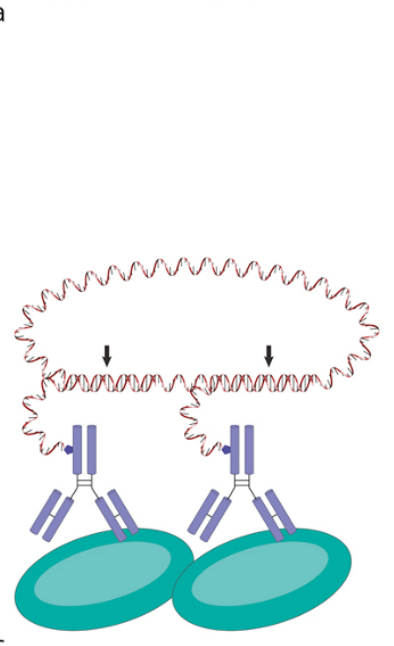

C

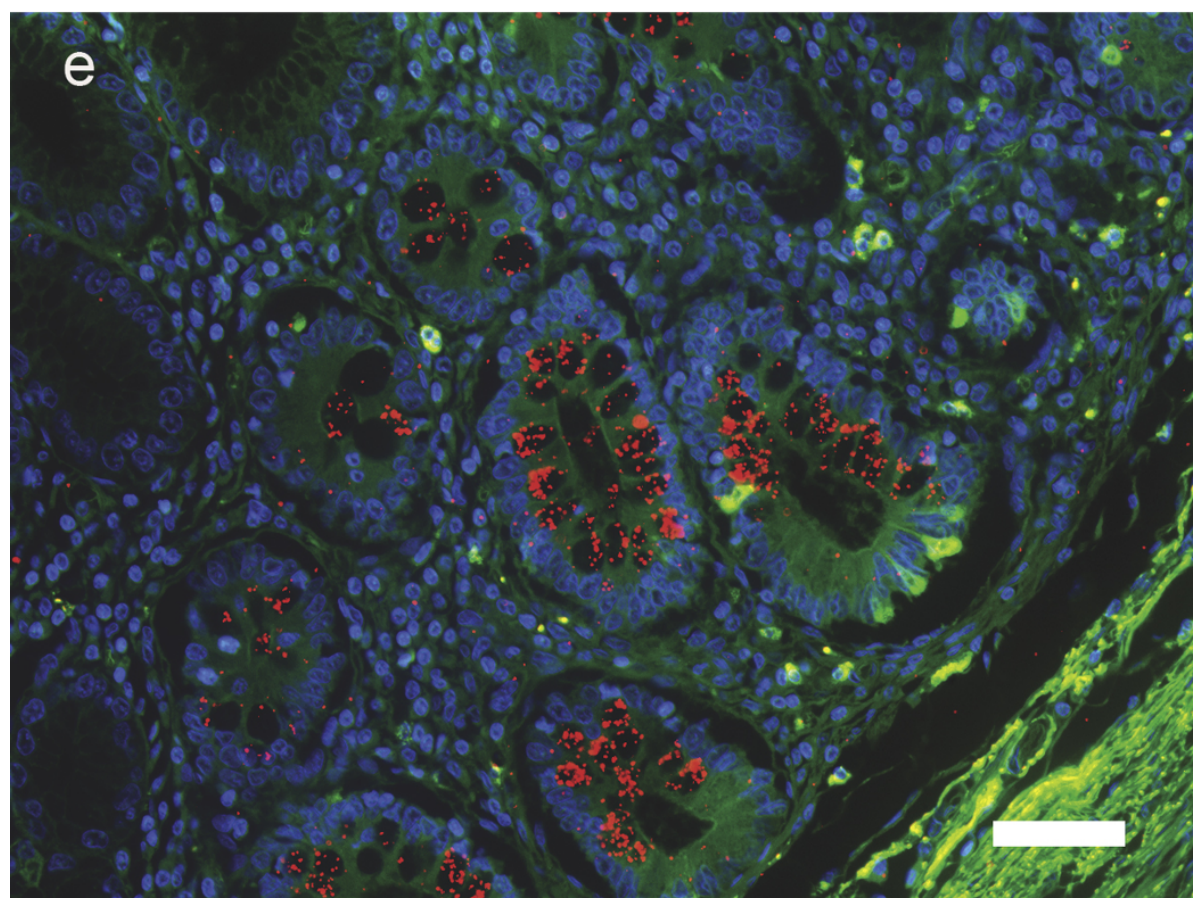

Figure 2 Detection of protein interactions with in situ PLA. a Proximal binding of PLA-probes to interacting proteins will guide the $\mathbf{b}$ hybridization of circularization probes, $\mathbf{c}$ allowing them to be connected by ligation (arrows) and thereby creating a circular reporter molecule of the protein interaction. $\mathbf{d}$ The oligonucleotide on one of the PLA-probes will then act as primer for RCA to generate an RCA product that will be an elongation of the PLA-probe. Fluorescence-labeled detection oligonucleotides are then used to visualize the RCA-product. e Detection of Mucin 2 glycosylation (Sialyl-Tn) (red dots) in formalin-fixed paraffin-embedded tissue sections from intestinal metaplasia [49]. Nuclei are counterstained with Hoechst 33342 (blue), using the autofluorescence of the tissue (green) to visualize the histology, scale bar equals $50 \mu \mathrm{m}$. 
an RCA product type originating from a circularization oligonucleotide used at a lower concentration, which will be reported by its respective other fluorophore-labeled detection oligonucleotide. By viewing the type of RCA products that are within the optimal dynamic range for each cell, it is possible to simultaneously measure in situ PLA targets in tissue sections containing cells with both high and low levels [51].

\section{Combined visualization of nucleic acids and proteins}

For visualization of interactions between protein and nucleic acid (PNI), to visualize e.g. epigenetic status of individual genomic sequences, things get more complicated as probes targeting both types of biomolecules are needed. To detect a specific DNA-sequence and its colocalization with a particular protein, immunofluorescence (IF) and FISH can be combined to form methods named immuno-DNA FISH and immuno-RNA FISH [52-54]. The protein of interest is targeted with fluorescencelabeled antibodies, while DNA is denatured and the target sequence detected by hybridization of hapten-labeled detection oligonucleotides. These haptens can in turn be detected by IF through utilizing antibodies targeting the hapten label. By superimposing the different fluorescence channels, co-localization of the nucleic acid sequence and the protein can be detected and visualized in situ. Although immuno-DNA/RNA FISH presents a valuable tool for investigation of PNIs in situ, spatial resolution is restricted to the resolution of the microscope used and proteins can only be detected if they are expressed at a certain level or above for IF to distinguish them over background fluorescence. Furthermore, the method cannot distinguish between highly homologous sequences as it is based on hybridization of a detection oligonucleotide. To increase the selectivity, enabling detection of protein-DNA interaction with an SNP-resolution, we developed a method that is based on padlock probes to target the DNA. Upon ligation, the padlock probe is converted into a circularization probe that will be utilized to survey the vicinity for bound PLA-probes. In order to prevent binding of the padlock probe to free PLA-probe, due to sequence complementarity, the padlock probe was equipped with hairpin structures that would hide the complementary sequences during PLA-probe staining. After washes the padlock probes can be activated, by degrading the uracil containing hairpins, thereby enabling hybridization to the bound PLA-probes [55]. After ligation the amplification and detection can be performed as for conventional in situ PLA. With this method we were able to detect proximity between histone $\mathrm{H} 3$ and Alu-repeats in human cells. Although the method was demonstrated to be highly selective in discriminating between closely homologous sequences (human and mouse Alu-repeats), it suffers from poor efficiency in targeting genomic DNA. Further efforts to make genomic DNA more accessible, i.e. by alternative fixations and sample pretreatments, are required to enable studies of single copy genes.

\section{The prospect of personalized medicine}

Although both padlock probes and in situ PLA are recently developed methods, we have high hopes that they within a near future will be adopted for routine diagnostics, just as IHC and FISH has become. A big advantage with in situ assays is that they do not require sophisticated and expensive equipment, both assays based on padlock probes and in situ PLA can be used with standard epifluorescence microscopes or, if the RCA-products are developed into chromogenic signals, even brightfield microscopes [50]. In situ analysis with padlock probes can be a rapid test for detection of recurrent mutations that might be useful to guide e.g. therapy choice, and the ability to genotype individual cells in a tumor provides the means to determine clonal evolution. To evaluate the functional consequences different mutations will have on a cell, in situ PLA can be used to determine how these mutations effects the proteins ability to interact with its partners. As mutations often occur at different proteins in a signaling pathway, by monitoring downstream hubs in signaling pathways all mutations with a similar consequence will be detected. In addition, as in situ PLA can be used on both cultured cells and tissue sections, the same assay can be used for drug development [56], diagnosis, selection of therapy and to monitor therapy response.

Considering the complexity of a tumor, the key factor for successful diagnostic assays lies in to what extent they can provide a coherent view of the cellular processes governing the growth and survival of the cancer cells. Methods to determine activity status of proteins, to analyze nucleic acids with a higher resolution and to determine interactions between these types of biomolecules at a single cell level will most likely be pivotal for future molecular pathology as they provide a tool to address cell-cell communication in the complex microenvironment of cancer. By understanding these processes, along with the ability for better characterization of individual patients, new routes for personalized treatment strategies will open up. Although cancer cells become addicted to the growth promoting signals derived from a mutated signaling pathway they still possess the ability to respond to other signals. The heterogeneity within the clone, and contacts with different cells and microenvironment of each individual cancer cells, provide escaperoutes when drugs are used to target one deregulated pathway. Single agent treatment strategies would thus probably have very limited success in eradicating all malignant cells within a patient. Hence, other growth and survival promoting 
pathways must be analyzed in addition to the ones altered by a mutation. An inherent restriction of single cell analyses is that the possibility to perform repetitive analysis on the same cell is limited. Because of this, multiplexed analyses are requested in order to obtain as much information as possible from each cell, and we have therefore been working on approaches to multiplex in situ PLA (Leuchowius et al., submitted). As padlock probes and in situ PLA utilize the same read-out-visualization of RCA products-it is possible to combine these methods for simultaneous detection of proteins and nucleic acids [57]. This provides a tool for investigation of active signaling both at the levels of signal transduction and expression of target genes.

As discussed previously, the interplay between the different cell types in a tumor will very likely have a major role on the outcome of all individual cancers and methods that identify patients with an adverse tumor cell communication will be important for prediction of disease and selection of therapy. The aberrant vessels formed by tumor angiogenesis may have a larger role in promoting growth of cancers than supporting them with nutrients and oxygen (something that they actually are deficient in), as the loosely assembled vessel walls facilitate intra- and extravasation of cancer cells. This will increase the possibility that CTC home to adjacent sites rather than forming distal metastasis. The hypoxic environment, due to insufficient organization of the tumor vessels, induces EMT that provides the cancer cells with a more motile phenotype. Both padlock probes and in situ PLA will very likely have a substantial role in elucidating the mechanisms involved in the cellular communications in tumors, something that then can be adopted for personalized medicine to predict disease progression, and in development and selection of therapy to restore the tumor microenvironment to a state that does not promote cancer growth. Albeit in situ PLA analysis of epigenetic alterations of individual promoters is not feasible at the moment, improvement in efficiency of the padlock-based in situ PLA [55] might soon provide a tool to investigate epigenetic reprogramming of cancer cells during transitions between epithelial and mesenchymal phenotypes.

Methods for analysis of cancer microenvironments will most likely have a large impact on personalized medicine and, although still early, the methods described herein may provide new opportunities to better predict disease progression and therapy response.

\section{Conflict of interest}

M.N. owns shares in the company Olink Biosciences that commercializes the PLA and padlock probe technologies.

\section{Acknowledgements}

We would like to thank Tim Conze for providing microscope image of in situ PLA. This work was sponsored by grants from Swedish Foundation for Strategic Research (SSF), Swedish Governmental Agency for Innovation Systems (Vinnova), Knut and Alice Wallenberg Foundation and the European Community's $7^{\text {th }}$ Framework Program (FP7/2007-2013), under grant agreement $n^{\circ} 259796$ (DiaTools).

Received: 20 December 2011 Accepted: 1 February 2012 Published: 22 March 2012

\section{References}

1. Vogelstein B, Fearon ER, Hamilton SR, Kern SE, Preisinger AC, Leppert M, et al: Genetic alterations during colorectal-tumor development. N Engl $J$ Med 1988, 319(9):525-32.

2. Sjöblom T, Jones S, Wood LD, Parsons DW, Lin J, Barber TD, et al: The consensus coding sequences of human breast and colorectal cancers. Science 2006, 314(5797):268-74.

3. Feinberg AP, Ohlsson R, Henikoff $S$ : The epigenetic progenitor origin of human cancer. Nat Rev Genet 2006, 7(1):21-33.

4. Illmensee K, Mintz B: Totipotency and normal differentiation of single teratocarcinoma cells cloned by injection into blastocysts. Proc Natl Acad Sci USA 1976, 73(2):549-53.

5. Mintz B, Illmensee K: Normal genetically mosaic mice produced from malignant teratocarcinoma cells. Proc Natl Acad Sci USA 1975, 72(9):3585-9.

6. Hentze H, Soong PL, Wang ST, Phillips BW, Putti TC, Dunn NR: Teratoma formation by human embryonic stem cells: Evaluation of essential parameters for future safety studies. Stem Cell Res 2009.

7. Lensch MW, Schlaeger TM, Zon LI, Daley GQ: Teratoma formation assays with human embryonic stem cells: a rationale for one type of humananimal chimera. Cell Stem Cell 2007, 1(3):253-8.

8. Müller FJ, Goldmann J, Löser P, Loring JF: A call to standardize teratoma assays used to define human pluripotent cell lines. Cell Stem Cell 2010, 6(5):412-4

9. Bissell MJ, Hines WC: Why don't we get more cancer? A proposed role of the microenvironment in restraining cancer progression. Nat Med 2011, 17(3):320-9.

10. Rich AR: On the frequency of occurrence of occult carcinoma of the prostrate. 1934. Int J Epidemiol 2007, 36(2):274-7.

11. Sakr W, Haas G, Cassin B, Pontes J, Crissman J: The frequency of carcinoma and intraepithelial neoplasia of the prostate in young male patients. $J$ Urol 1993, 150(2 Pt 1):379.

12. Hanahan D, Weinberg RA: Hallmarks of cancer: the next generation. Cell 2011, 144(5):646-74.

13. Grivennikov SI, Greten FR, Karin M: Immunity, inflammation, and cancer. Cell 2010, 140(6):883-99.

14. Kroemer G, Pouyssegur J: Tumor cell metabolism: cancer's Achilles' heel. Cancer Cell 2008, 13(6):472-82.

15. Liguori M, Solinas G, Germano G, Mantovani A, Allavena P: Tumorassociated macrophages as incessant builders and destroyers of the cancer stroma. Cancers 2011, 3(4):3740-61.

16. Schäfer $M$, Werner $S$ : Cancer as an overhealing wound: an old hypothesis revisited. Nat Rev Mol Cell Biol 2008, 9(8):628-38.

17. Potente $M$, Gerhardt $H$, Carmeliet P: Basic and therapeutic aspects of angiogenesis. Cell 2011, 146(6):873-87.

18. Comen E, Norton L, Massague J: Clinical implications of cancer selfseeding. Nat Rev Clin Oncol 2011, 8(6):369-77.

19. Huh SJ, Liang S, Sharma A, Dong C, Robertson GP: Transiently entrapped circulating tumor cells interact with neutrophils to facilitate lung metastasis development. Cancer Research 2010, 70(14):6071-82.

20. Chen $Q$, Zhang XH, Massague J: Macrophage binding to receptor VCAM-1 transmits survival signals in breast cancer cells that invade the lungs. Cancer Cell 2011, 20(4):538-49.

21. Ferrara N: Pathways mediating VEGF-independent tumor angiogenesis. Cytokine Growth Factor Rev 2010, 21(1):21-6.

22. Bhowmick NA, Neilson EG, Moses HL: Stromal fibroblasts in cancer initiation and progression. Nature 2004, 432(7015):332.

23. Klopp AH, Gupta A, Spaeth E, Andreeff M, Marini F: Concise review: dissecting a discrepancy in the literature: do mesenchymal stem cells support or suppress tumor growth? Stem Cells 2011, 29(1):11-9. 
24. Takebe N, Ivy SP: Controversies in cancer stem cells: targeting embryonic signaling pathways. Clin Cancer Res 2010, 16(12):3106-12.

25. Visvader JE, Lindeman GJ: Cancer stem cells in solid tumours: accumulating evidence and unresolved questions. Nat Rev Cancer 2008, 8(10):755-68.

26. Yang MH, Wu MZ, Chiou SH, Chen PM, Chang SY, Liu CJ, et al: Direct regulation of TWIST by HIF-1alpha promotes metastasis. Nat Cell Biol 2008, 10(3):295-305.

27. Wu MZ, Tsai YP, Yang MH, Huang CH, Chang SY, Chang CC, et al: Interplay between HDAC3 and WDR5 is essential for hypoxiainduced epithelialmesenchymal transition. Mol Cell 2011, 43(5):811-22.

28. Daniel VC, Marchionni L, Hierman JS, Rhodes JT, Devereux WL, Rudin CM, et al: A primary xenograft model of small-cell lung cancer reveals irreversible changes in gene expression imposed by culture in vitro. Cancer Res 2009, 69(8):3364-73.

29. Li A, Walling J, Kotliarov Y, Center A, Steed ME, Ahn SJ, et al: Genomic changes and gene expression profiles reveal that established glioma cell lines are poorly representative of primary human gliomas. Mol Cancer Res 2008, 6(1):21-30.

30. Bauman JGJ, Wiegant J, Borst P, van Duijn P: A new method for fluorescence microscopical localization of specific DNA sequences by in situ hybridization of fluorochrome-labelled RNA. Exp Cell Res 1980, 128(2):485-90.

31. Nilsson M, Malmgren H, Samiotaki M, Kwiatkowski M, Chowdhary BP, Landegren U: Padlock probes: circularizing oligonucleotides for localized DNA detection. Science 1994, 265(5181):2085-8.

32. Nilsson M, Kreji K, Koch J, Kwiatkowski M, Gustavsson P, Landegren U: Padlock probes reveal single-nucleotide differences, parent of origin and in situ distribution of centromeric sequences in human chromosomes 13 and 21. Nat Genet 1997, 16(3):252-5.

33. Lizardi PM, Huang X, Zhu Z, Bray-Ward P, Thomas DC, Ward DC: Mutation detection and single-molecule counting using isothermal rolling-circle amplification. Nat Genet 1998, 19(3):225-32.

34. Blab GA, Schmidt T, Nilsson M: Homogeneous detection of single rolling circle replication products. Anal Chem 2004, 76(2):495-8.

35. Larsson C, Koch J, Nygren A, Janssen G, Raap AK, Landegren U, et al: In situ genotyping individual DNA molecules by target-primed rolling-circle amplification of padlock probes. Nat Methods 2004, 1(3):227-32.

36. Nilsson M, Barbany G, Antson DO, Gertow K, Landegren U: Enhanced detection and distinction of RNA by enzymatic probe ligation. Nat Biotechnol 2000, 18(7):791-3.

37. Nilsson M, Landegren $U$, Antson DO: Single-nucleotide sequence discrimination in situ using padlock probes. Curr Protoc Cytom 2001, Chapter 8, Unit 8.8.

38. Larsson C, Grundberg I, Söderberg O, Nilsson M: In situ detection and genotyping of individual mRNA molecules. Nat Methods 2010, 7(5):395-7.

39. Silahtaroglu A, Pfundheller H, Koshkin A, Tommerup N, Kauppinen S: LNAmodified oligonucleotides are highly efficient as FISH probes. Cytogenet Genome Res 2004, 107(1-2):32-7.

40. Silahtaroglu AN, Tommerup N, Vissing H: FISHing with locked nucleic acids (LNA): evaluation of different LNA/DNA mixmers. Mol Cell Probes 2003, 17(4):165-9.

41. Kurreck J, Wyszko E, Gillen C, Erdmann VA: Design of antisense oligonucleotides stabilized by locked nucleic acids. Nucleic Acids Res 2002, 30(9):1911-8.

42. Schermelleh L, Heintzmann R, Leonhardt $\mathrm{H}$ : A guide to superresolution fluorescence microscopy. J Cell Biol 2010, 190(2):165-75.

43. Selvin PR: The renaissance of fluorescence resonance energy transfer. Nat Struct Biol 2000, 7(9):730-4

44. Berezovska O, Ramdya P, Skoch J, Wolfe MS, Bacskai BJ, Hyman BT: Amyloid precursor protein associates with a nicastrin-dependent docking site on the presenilin 1-gamma-secretase complex in cells demonstrated by fluorescence lifetime imaging. J Neuroscience 2003, 23(11):4560-6.

45. Jones PB, Rozkalne A, Meyer-Luehmann M, Spires-Jones TL, Makarova A, Kumar ATN, et al: Two postprocessing techniques for the elimination of background autofluorescence for fluorescence lifetime imaging microscopy. J Biomed Opt 2008, 13:014008.

46. Söderberg O, Gullberg M, Jarvius M, Ridderstråle K, Leuchowius KJ, Jarvius J, et al: Direct observation of individual endogenous protein complexes in situ by proximity ligation. Nat Methods 2006, 3(12):995-1000.
47. Baan $B$, Pardali $E$, ten Dijke $P$, van Dam $H$ : In situ proximity ligation detection of C-Jun/AP-1 dimers reveals increased levels of c-Jun/Fra1 complexes in aggressive breast cancer cell lines in vitro and in vivo. Mol Cell Proteomics 2010, 9(9):1982.

48. Jarvius M, Paulsson J, Weibrecht I, Leuchowius K, Andersson AC, Wählby C, et al: In situ detection of phosphorylated platelet-derived growth factor receptor $\beta$ using a generalized proximity ligation method. Mol Cell Proteomics 2007, 6(9):1500.

49. Conze $\mathrm{T}$, Carvalho AS, Landegren U, Almeida R, Reis CA, David L, et al: MUC2 mucin is a major carrier of the cancer-associated sialyl-Tn antigen in intestinal metaplasia and gastric carcinomas. Glycobiology 2010, 20(2):199.

50. Zieba A, Wählby C, Hjelm F, Jordan L, Berg J, Landegren U, et al: Brightfield microscopy visualization of proteins and protein complexes by in situ proximity ligation with peroxidase detection. Clin Chem 2010, 56(1):99-110

51. Clausson CM, Allalou A, Weibrecht I, Mahmoudi S, Farnebo M, Landegren U, et al: Increasing the dynamic range of in situ PLA. Nat Methods 2011, 8(11):892-3.

52. Zirbel R, Mathieu U, Kurz A, Cremer T, Lichter P: Evidence for a nuclear compartment of transcription and splicing located at chromosome domain boundaries. Chromosome Res 1993, 1(2):93-106.

53. Brown K: Visualizing nuclear proteins together with transcribed and inactive genes in structurally preserved cells. Methods 2002, 26(1):10-8.

54. Brown KE, Guest SS, Smale ST, Hahm K, Merkenschlager M, Fisher AG: Association of transcriptionally silent genes with lkaros complexes at centromeric heterochromatin. Cell 1997, 91(6):845-54.

55. Weibrecht I, Gavrilovic M, Lindbom L, Landegren U, Wählby C, Söderberg O Visualising individual sequence-specific protein-DNA interactions in situ. N Biotechnol, doi:10.1016/j.nbt.2011.08.002.

56. Leuchowius KJ, Jarvius M, Wickström M, Rickardson L, Landegren U, Larsson R, et al: High content screening for inhibitors of protein interactions and post-translational modifications in primary cells by proximity ligation. Mol Cell Proteomics 2010, 9(1):178-83.

57. Weibrecht I, Grundberg I, Nilsson M, Söderberg O: Simultaneous visualization of both signaling cascade activity and end-point gene expression in single cells. PLoS One 2011, 6(5):e20148.

doi:10.1007/s13167-012-0140-3

Cite this article as: Clausson et al:: Methods for analysis of the cancer microenvironment and their potential for disease prediction, monitoring and personalized treatments. EPMA Journal 2012 3:7.

\section{Submit your next manuscript to BioMed Central and take full advantage of:}

- Convenient online submission

- Thorough peer review

- No space constraints or color figure charges

- Immediate publication on acceptance

- Inclusion in PubMed, CAS, Scopus and Google Scholar

- Research which is freely available for redistribution 